\title{
iStent trabecular micro-bypass stent for open-angle glaucoma
}

This article was published in the following Dove Press journal:

Clinical Ophthalmology

23 September 2014

Number of times this article has been viewed

\section{Kim Le \\ Hady Saheb}

Department of Ophthalmology, McGill University, Montreal, QC, Canada
Correspondence: Hady Saheb McGill University Health Center, 687 Pine Ave Ouest, Montreal, QC H3A IAI, Canada

$\mathrm{Tel}+\mathrm{I} 5 \mid 49341934$ ext 35763

$\mathrm{Fax}+15148431612$

Email hady.saheb@mcgill.ca
Abstract: Trabecular micro-bypass stents, commonly known as iStents, are micro-invasive glaucoma surgery (MIGS) devices used to treat open-angle glaucoma. Like other MIGS procedures that enhance trabecular outflow, the iStent lowers intraocular pressure (IOP) by creating a direct channel between the anterior chamber and Schlemm's canal. iStents are typically implanted at the time of phacoemulsification for patients with open-angle glaucoma and visually significant cataracts. This review summarizes the published data regarding the efficacy, safety, and cost considerations of trabecular micro-bypass stents. Most studies found statistically significant reductions in mean IOP and ocular medication use after combined phacoemulsification with single or double iStent implantation. The devices were found to be very safe, with a safety profile similar to that of cataract surgery. Complications were infrequent, with the most common complications being temporary stent obstruction or malposition, which resolved with observation or secondary procedures. Future studies are needed to evaluate long-term outcomes, patient satisfaction, cost effectiveness, and expanded indications.

Keywords: iStent, trabecular micro-bypass, glaucoma, MIGS, ab interno, surgery

\section{Introduction}

The worldwide prevalence of open-angle glaucoma is 60.5 million. ${ }^{1}$ Traditionally, treatment for glaucoma has included topical hypotensive medications, laser therapy, and surgery. Patient adherence to medication is suboptimal due to common side effects such as ocular surface irritation, ${ }^{2}$ difficulty of proper application, ${ }^{3}$ and financial considerations. ${ }^{4}$ Poor control of glaucoma despite medications and adjuvant laser trabeculoplasty is typically managed with surgical therapy. The traditional full-thickness glaucoma surgeries of trabeculectomy and glaucoma drainage devices effectively lower intraocular pressure (IOP); however, serious complications, including hypotony, choroidal effusions, suprachoroidal hemorrhage, corneal edema, diplopia, and long-term risks of endophthalmitis, accompany these procedures. ${ }^{5}$ In recent years, the development of a new class of procedures, termed micro-invasive glaucoma surgery (MIGS), has raised excitement within the glaucoma community by offering an alternative form of effective IOP reduction associated with lower medication burden and complication rates. ${ }^{6}$ Our review focuses on trabecular micro-bypass stents, one of the most widely used MIGS devices. A PubMed search for 'iStent' revealed 26 articles. Each of these full-text articles was reviewed. Secondary searches for 'trabecular bypass' and 'MIGS' identified additional relevant articles. Randomized controlled trials (RCTs), case series, review articles, and cost studies were cited in this paper.

\section{Design}

The iStent ${ }^{\circledR}$ (Glaukos Corporation, Laguna Hills, CA, USA) is a first-generation trabecular micro-bypass product. It is a heparin-coated non-ferromagnetic, titanium stent connecting the anterior chamber directly to Schlemm's canal. With dimensions 
of $1 \mathrm{~mm}$ in length and $0.3 \mathrm{~mm}$ in height, the iStent is the smallest implantable medical device approved for use in humans by the US Food and Drug Administration.

Using gonioscopy, the device can be visualized within Schlemm's canal and in the anterior chamber, allowing aqueous to flow between these two spaces. Perpendicular to the inlet, the longer pointed end facilitates entry into Schlemm's canal, and the three retention arches secure its position. ${ }^{7,8}$ The half-cylinder profile with an open posterior wall prevents blockage or fibrosis over the tip ${ }^{8,9}$ (Figures 1, 2, and 3). The inserter consists of 26-gauge tubing and four finger extensions. This design facilitates device implantation via a clear corneal incision and ab interno approach under gonioscopy. ${ }^{10}$ These extensions also allow re-grasping of the device if further manipulation or repositioning is necessary.

A second-generation model, the iStent inject ${ }^{\circledR}$ (Glaukos Corporation, Laguna Hills, CA, USA), has been certified in Europe. ${ }^{11}$ It is similarly composed of titanium, and is only 360 microns in length, smaller in size than the first-generation iStent. Its shape is characterized by an apical head, 230 microns in width, containing four inlets for the passage of aqueous. The narrow body contains the lumen and sits within the trabecular meshwork. A flange secures the device on the inner wall of the meshwork, allowing aqueous to exit into Schlemm's canal (Figures 4, 5, and 6). A 23-gauge stainless steel insertion sleeve covers the injector, which comes preloaded with two stents. ${ }^{12}$ This newer model has the advantage of easier surgical technique; no sideways sliding of the stent is required for positioning, and two devices can be implanted with a single inserter without exiting and re-entering the eye.

\section{Surgical technique}

If combined with phacoemulsification, iStent implantation is performed using the same clear corneal incision. If the iStent is implanted without phacoemulsification, a clear corneal incision of 1.5-2 $\mathrm{mm}$ is performed temporally. An ophthalmic viscoelastic device (OVD) is used to inflate the anterior chamber and widen the nasal angle. After rotating the head

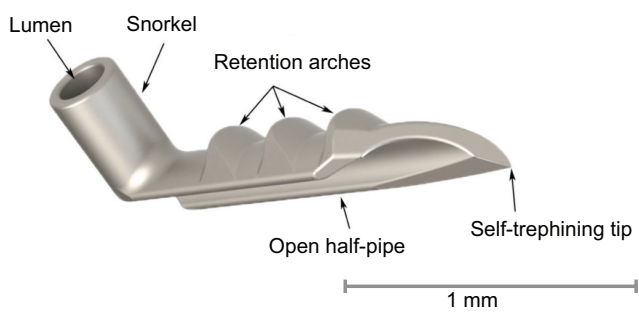

Figure I First-generation iStent.

Notes: Copyright (C 2014. Dove Medical Press. Adapted from Hunter KS, Fjield T, Heitzmann H, Shandas R, Kahook MY. Characterization of micro-invasive trabecular bypass stents by ex vivo perfusion and computational flow modeling. Clin Ophthalmol. 2014;8:499-506..$^{14}$

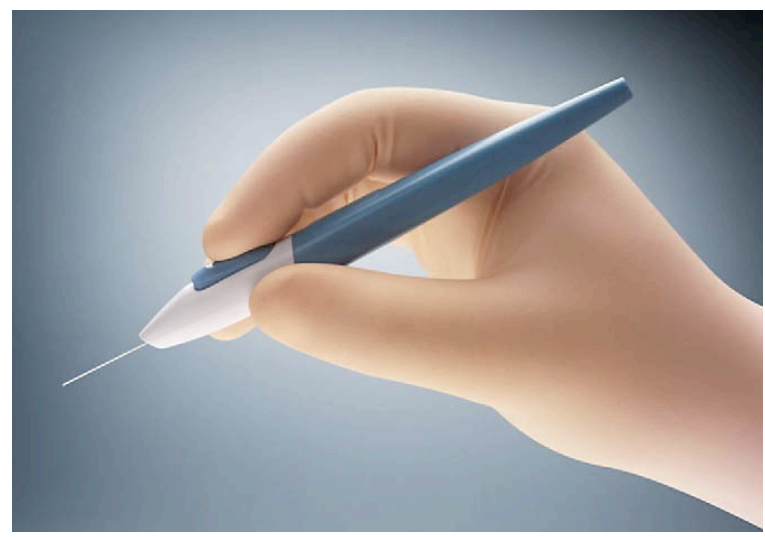

Figure 2 Applicator for the first-generation iStent.

Notes: Reprinted from Minckler DS, Hill RA. Use of novel devices for control of intraocular pressure. Exp Eye Res. 2009;88(4):792-798. Copyright (C) 2009, with permission from Elsevier. ${ }^{10}$

of the patient $30^{\circ}$ away from the surgeon, and the oculars of the surgical microscope $30^{\circ}$ towards the surgeon, the iStent inserter crosses the anterior chamber and approaches the nasal angle under direct gonioscopic view. The iStent punctures the trabecular meshwork and slides into Schlemm's canal. The OVD is then rinsed out of the anterior chamber unless phacoemulsification follows.

The authors' preference is to perform iStent implantation prior to phacoemulsification because the view through the cornea is more likely to be clear, the sclera is less hydrated and its rigidity is more favorable for iStent implantation, and potential complications of phacoemulsification do not affect iStent implantation. Some surgeons favor performing iStent implantation following phacoemulsification because the angle is wider and the anterior capsule is less at risk of inadvertent breach. However, it is worth noting that, in patients with open-angle glaucoma, a significant opening of the angle can be achieved with OVD prior to phacoemulsification.

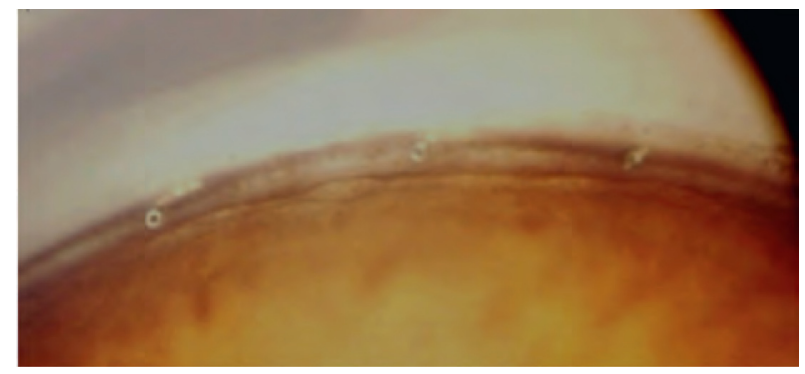

Figure 3 Gonioscopic view of the angle with three implanted iStents into the trabecular meshwork.

Notes: Copyright (C) 2012. Reproduced with permission from Lippincott Williams and Wilkins/Wolters Kluwer Health, from: Saheb H, Ahmed II. Micro-invasive glaucoma surgery: current perspectives and future directions. Curr Opin Ophthalmol. 2012;23(2):96-104. ${ }^{6}$ Promotional and commercial use of the material in print, digital or mobile device format is prohibited without the permission from the publisher Lippincott Williams \& Wilkins. Please contact journalpermissions@lww.com for further information. 


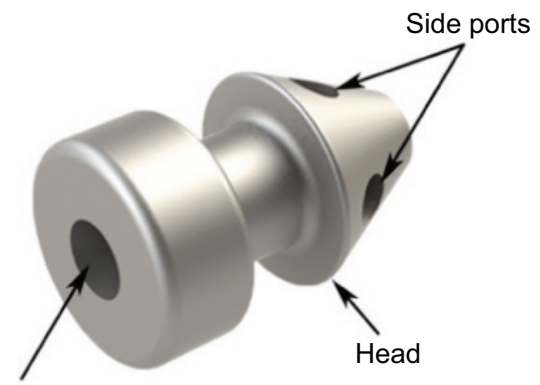

Central lumen

$1 \mathrm{~mm}$

Figure 4 Second-generation iStent inject.

Notes: Copyright (C) 2014. Dove Medical Press. Adapted from Hunter KS, Fjield T, Heitzmann H, Shandas R, Kahook MY. Characterization of micro-invasive trabecular bypass stents by ex vivo perfusion and computational flow modeling. Clin Ophthalmol. 2014;8:499-506. ${ }^{14}$

Other aspects of iStent implantation that can optimize the success of the procedure are important to consider. Wounds should be slightly anterior to the limbus to avoid limbal vessels and bleeding that could impair the quality of the gonioscopic view. OVD under-filling could contribute to a soft eye that easily develops corneal folds under gonioscopic view. However, an overfilled eye can lead to a flattened Schlemm's canal. The approach to entry into Schlemm's canal should be similar to venipuncture: a sharper initial approach to puncture the trabecular meshwork followed by a flattened approach to slide into Schlemm's canal without abutting against the outer wall of Schlemm's canal. Like venipuncture, there should be no resistance throughout the insertion of the iStent. Any torque of the eye during the insertion of the iStent is likely due to the iStent abutting against the outer wall of Schlemm's canal (sclera); the authors suggest backing up the iStent and flattening the approach of the iStent in that case until no torque or resistance occurs during the insertion. Furthermore, any
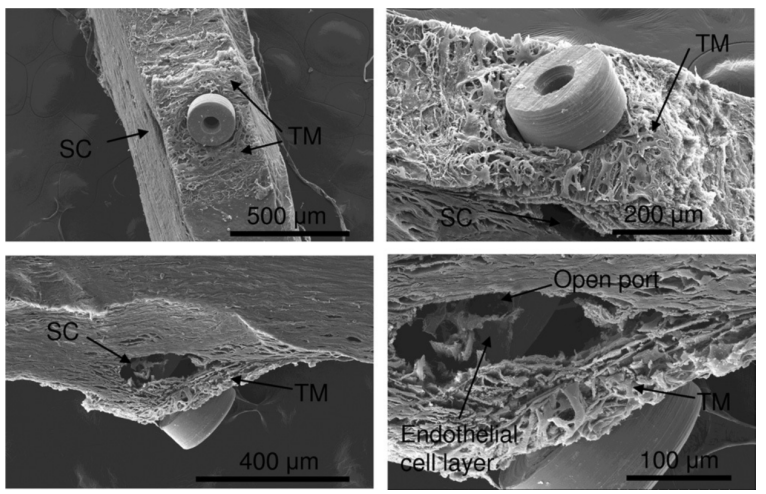

Figure 5 Various scanning electron microscope views of the iStent inject positioned within the trabecular meshwork (TM) and Schlemm's canal (SC) in human anterior segment culture.

Notes: Reprinted from Bahler CK, Hann CR, Fjield T, Haffner D, Heitzmann H, Fautsch MP. Second-generation trabecular meshwork bypass stent (iStent inject) increases outflow facility in cultured human anterior segments. Am J Ophthalmol. 2012;153(6): 1206-1213. Copyright (C) 2012, with permission from Elsevier.'2
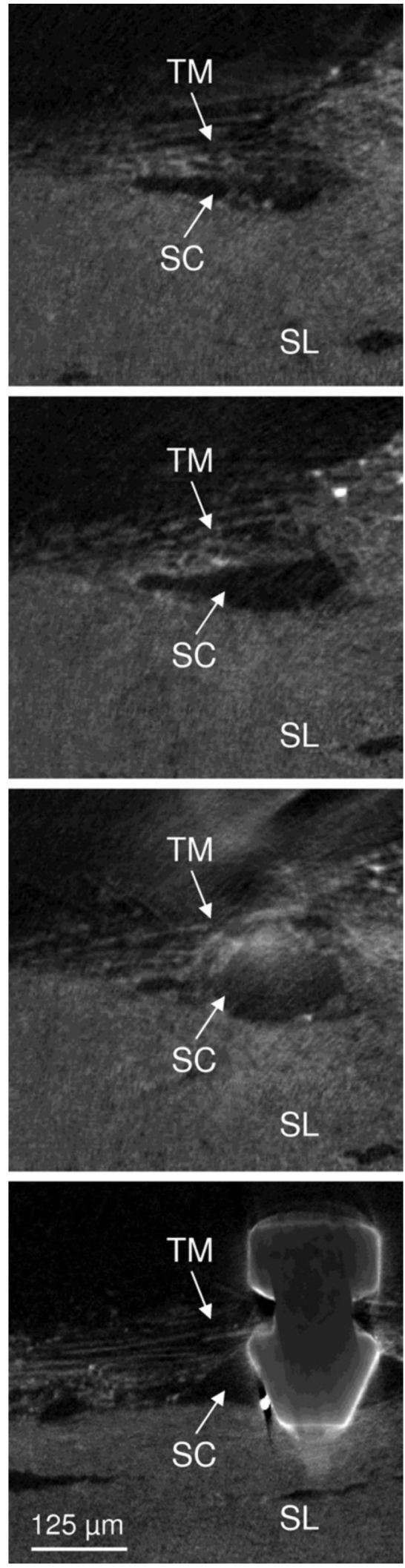

Figure 6 Three-dimensional micro-computed tomography views showing dilatation of Schlemm's canal (SC) after implantation of the iStent inject, in relation to the trabecular meshwork (TM) and sclera (SL).

Notes: Reprinted from Bahler CK, Hann CR, Fjield T, Haffner D, Heitzmann H, Fautsch MP. Second-generation trabecular meshwork bypass stent (iStent inject) increases outflow facility in cultured human anterior segments. Am J Ophthalmol. 2012;153(6): 1206-1213. Copyright (C 2012, with permission from Elsevier. ${ }^{12}$ 
intracameral bleeding can be cleared using an OVD. Blood reflux from the iStent is normal and can signify that the trabecular outflow system is patent. Prior to moving on to the next step of surgery, it is critical to ensure the proper positioning of the iStent within Schlemm's canal.

The implantation of the second-generation microbypass stent (iStent inject) shares many features of the first-generation stent. The difference lies in the uni-dimensional aspect of its insertion. Once the trabecular meshwork is punctured by the sharp tip of the inserter, the stent is released into Schlemm's canal, so that sideways sliding is not required. Furthermore, two stents can be implanted with the same inserter.

\section{Mechanism of action}

By creating and maintaining a channel between the aqueous in the anterior chamber and Schlemm's canal, the iStent bypasses the site of highest resistance to aqueous outflow: the juxtacanalicular trabecular meshwork. ${ }^{13}$ Flow rates and resistance through the devices have been studied in ex vivo models. Hunter et $\mathrm{al}^{14}$ found that a single iStent reduced total outflow resistance by $30 \%$ and IOP by $6 \mathrm{mmHg}$, using a human whole eye perfusion model, computational fluid dynamics, and the Hagen-Poiseuille equation. Two stents decreased resistance by $44 \%$ and IOP by $8.9 \mathrm{mmHg}$ from baseline. ${ }^{14}$ Using anterior segment perfusion models, Bahler et $\mathrm{al}^{15}$ found similar drops in IOP of $6.1 \mathrm{mmHg}$ with one iStent and $9.7 \mathrm{mmHg}$ with two. They repeated the protocol for the iStent inject, which increased outflow facility from 0.16 to $0.38 \mu \mathrm{L} / \mathrm{min} / \mathrm{mmHg}$ with one device and $0.78 \mu \mathrm{L} / \mathrm{min} / \mathrm{mmHg}$ with two. ${ }^{12}$ These results suggested that the IOP-lowering effect could be titrated with the number of implants.

\section{Efficacy}

A number of clinical studies from the past decade have examined the efficacy of the iStent and iStent inject. A summary of these RCTs and case series can be found in Table 1. Looking at the three RCTs and seven case series that examined the efficacy of single or multiple iStents with or without phacoemulsification, IOP reduction ranged from $16 \%$ to $33 \%$, and medication reduction ranged from 0.5 to 2.0 agents, as seen in Table 1 . With respect to the iStent inject with or without phacoemulsification, only two case series have been published. Listed in Table 1, both studies implanted two stents and found that IOP decreased almost $40 \%$ and medication decreased by one agent. The individual studies are described in the following sections.

Table I Summary of trabecular micro-bypass clinical studies

\begin{tabular}{|c|c|c|c|c|c|c|c|c|c|}
\hline Authors & Design & Site & $\mathbf{n}$ & $\begin{array}{l}\text { Glaucoma } \\
\text { type }\end{array}$ & Procedure & Years & IOP reduction & $\begin{array}{l}\text { Medication } \\
\text { reduction }\end{array}$ & Washout \\
\hline \multicolumn{10}{|l|}{ iStent } \\
\hline Craven et al ${ }^{16}$ & RCT & USA & 116 & $\begin{array}{l}\text { POAG, PDG, } \\
\text { PXG }\end{array}$ & I stent + phaco & 2 & $8 \mathrm{mmHg}, 33 \%$ & 1.3 & Yes \\
\hline Samuelson et $\mathrm{al}^{17}$ & RCT & USA & 111 & $\begin{array}{l}\text { POAG, PDG, } \\
\text { PXG }\end{array}$ & I stent + phaco & 1 & $8 \mathrm{mmHg}, 33 \%$ & 1.4 & Yes \\
\hline $\mathrm{Fea}^{18}$ & RCT & Italy & 12 & POAG & I stent + phaco & 1.25 & $3 \mathrm{mmHg}, 17 \%$ & 1.6 & Yes \\
\hline Fernández-Barrientos et al ${ }^{19}$ & RCT & Spain & 17 & POAG, OHT & 2 stents + phaco & I & $7 \mathrm{mmHg}, 27 \%$ & I.I & Yes \\
\hline Belovay et $\mathrm{a}^{25}$ & Series & Canada & 53 & $\begin{array}{l}\text { POAG, PXE, } \\
\text { mixed }\end{array}$ & $2-3$ stents + phaco & I & $4 \mathrm{mmHg}, 20 \%$ & 2.0 & No \\
\hline Spiegel et $\mathrm{al}^{22}$ & Series & Europe & 48 & $\begin{array}{l}\text { POAG, PDG, } \\
\text { PXG }\end{array}$ & I stent + phaco & I & $4 \mathrm{mmHg}, 18 \%$ & 1.2 & No \\
\hline Arriola-Villalobos et $\mathrm{a}^{28}$ & Series & Spain & 19 & $\begin{array}{l}\text { POAG, PDG, } \\
\text { PXG }\end{array}$ & I stent + phaco & 5 & $3 \mathrm{mmHg}, 16 \%$ & 0.5 & No \\
\hline Vandewalle et $\mathrm{al}^{27}$ & Series & Belgium & 10 & OAG, PXG & I stent \pm phaco & I & $4 \mathrm{mmHg}, 19 \%$ & 1.0 & No \\
\hline Patel et $\mathrm{a}^{23}$ & Series & UK & 44 & $\begin{array}{l}\text { POAG, PXG, } \\
\text { recession }\end{array}$ & I stent \pm phaco & 0.5 & $4 \mathrm{mmHg}, 2 \mathrm{I} \%$ & 1.2 & No \\
\hline Buchacra et a ${ }^{26}$ & Series & Spain & 10 & $\begin{array}{l}\text { PDG, PXG, } \\
\text { trauma, steroid }\end{array}$ & I stent & 1 & $7 \mathrm{mmHg}, 27 \%$ & I.I & No \\
\hline \multicolumn{10}{|l|}{ iStent inject } \\
\hline Arriola-Villalobos et $\mathrm{al}^{24}$ & Series & Spain & 20 & $\begin{array}{l}\text { POAG, PXG, } \\
\text { OHT }\end{array}$ & 2 stents + phaco & I & $9 \mathrm{mmHg}, 36 \%$ & 1.0 & Yes \\
\hline Voskanyan et al" & Series & Europe & 99 & $\begin{array}{l}\text { POAG, PDG, } \\
\text { PXG }\end{array}$ & 2 stents & I & $10 \mathrm{mmHg}, 40 \%$ & 2.0 & Yes \\
\hline
\end{tabular}

Note: The numerical value listed under 'Medication Reduction' represents the decrease in mean number of hypotensive agents used after the intervention.

Abbreviations: IOP, intraocular pressure; OAG, open-angle glaucoma; OHT, ocular hypertension; PDG, pigment-dispersion glaucoma; phaco, phacoemulsification; POAG, primary open-angle glaucoma; PXG, pseudoexfoliation glaucoma; RCT, randomized controlled trial. 
Trabecular stent with phacoemulsification: RCTs

The efficacy of the iStent was studied in a prospective, multicenter RCT that measured IOP reduction and medication use in patients with concurrent cataracts and mild to moderate open-angle glaucoma. ${ }^{16,17}$ Across 29 centers, 240 eyes with cataract and primary open angle glaucoma (POAG), pseudoexfoliation glaucoma (PXG), or pigment-dispersion glaucoma (PDG) were randomized to phacoemulsification alone (control group) or combined phacoemulsification with one iStent (treatment group). Inclusion criteria included best corrected visual acuity (BCVA) of 20/40 or worse, IOP $\leq 24 \mathrm{mmHg}$ while taking one to three ocular hypotensive medications, and IOP between 22 and $36 \mathrm{mmHg}$ after washout of glaucoma medications. The primary efficacy outcome was the proportion of patients with month $12 \mathrm{IOP} \leq 21 \mathrm{mmHg}$ without medications. This was achieved by $72 \%$ of the iStent group versus $50 \%$ of the phacoemulsification alone group after 1 year $(P<0.001)$. The secondary outcome was unmedicated month 12 IOP reduction $\geq 20 \%$ from baseline, which was seen in $66 \%$ of treated versus $48 \%$ of control eyes $(P<0.003)$. The mean reduction in IOP was $30 \%$ or $8 \mathrm{mmHg}$ in both groups at 12 months. This was expected because the protocol called for subjects to be managed to an IOP of $21 \mathrm{mmHg}$ or lower regardless of treatment group, through the use of glaucoma medications. At 12 months, Samuelson et al found that $15 \%$ of patients who received the iStent with phacoemulsification were using medications compared with $35 \%$ of controls $(P=0.001)^{17}$ (Table 1 , row 2 ).

Results at 2 years by Craven et al showed relatively similar outcomes, as seen in Table 1, row 1. ${ }^{16}$ Although the study was neither designed nor statistically powered to include 2-year efficacy endpoints, $61 \%$ of treated versus $50 \%$ of control eyes had month 24 IOP $\leq 21 \mathrm{mmHg}$ without medications $(P<0.036)$. A total of $53 \%$ of treated eyes compared with $44 \%$ of control eyes had unmedicated month 24 IOP reduction $\geq 20 \%(P<0.090)$. Overall mean IOP in the stent group was $17.0 \mathrm{mmHg}$ after 1 year and $17.1 \mathrm{mmHg}$ after 2 years, whereas the control group had $17.0 \mathrm{mmHg}$ and $17.8 \mathrm{mmHg}$ during the same time period. However, the difference in ocular hypotensive medication use was no longer statistically significant between groups after 24 months.

Prior to that study, $\mathrm{Fea}^{18}$ performed a prospective, doublemasked RCT on 36 Italian patients with cataract and POAG. Inclusion criteria included IOP $>18 \mathrm{mmHg}$, receiving at least one medication, and vision no better than 20/80. Patients received either phacoemulsification alone or in combination with a single iStent (Table 1, row 3). IOP dropped from 17.9 to $14.8 \mathrm{mmHg}$ in the combined group and from 17.3 to $15.7 \mathrm{mmHg}$ in controls at 15 months $(P<0.031)$. This unique study design included a washout of medications at 15 months to remove the confounding effect of glaucoma medications. Following washout at 16 months, IOP was $16.6 \mathrm{mmHg}$ in treated versus $19.2 \mathrm{mmHg}$ in control eyes $(P<0.042)$. The mean number of medications, as well as the proportion of patients receiving medications, was significantly lower in the stent group $(P<0.007)$.

A different prospective RCT used fluorophotometry to compare aqueous flow, trabecular outflow facility, and IOP reduction in 33 eyes with cataract and open-angle glaucoma or ocular hypertension (OHT).$^{19}$ This time, two iStents were inserted, along with phacoemulsification, in the treatment arm and compared with phacoemulsification alone. While there were no significant changes in flow following intervention, facility increased $275 \%$ and $46 \%$ in the treatment and control groups, respectively, $(P<0.02)$ after 12 months. FernándezBarrientos et $\mathrm{l}^{19}$ found a corresponding mean IOP reduction of $6.6 \mathrm{mmHg}$ versus $3.9 \mathrm{mmHg}(P<0.002)$ and medication use of 0.0 vs $0.7(P<0.007)$ in the stent versus phacoemulsification groups, respectively (Table 1 , row 4$)$. This represented a larger relative treatment effect than in single stent plus phacoemulsification studies, which Augustinus and Zeyen ${ }^{20}$ estimated to lower IOP by $3-4 \mathrm{mmHg}$ on average in a review.

\section{Trabecular stent with phacoemulsification: case series}

Published case series have reported comparable reductions in IOP and medication use after combined phacoemulsification and single stent implantation. In a much earlier study, Spiegel et $\mathrm{al}^{21,22}$ performed combined procedures in 58 patients with POAG, PXG, and PDG across four European centers. ${ }^{21,22}$ At baseline, mean IOP was $21.5 \mathrm{mmHg}$ on 1.5 medications. At 6 months, IOP dropped by $25 \%$ or $5.7 \mathrm{mmHg}(P<0.001)$, and medications decreased by 1.0 agents $(P<0.001) .{ }^{21}$ After 1 year, the treatment effect diminished slightly, with IOP reduction of $18 \%$ or $4.4 \mathrm{mmHg}$ from baseline $(P<0.001)$; however, the treatment effect of adequate control without medications remained stable, with a reduction of 1.2 agents $(P<0.001)$ as seen in Table 1, row 6.22 Two-year results are pending. A more recent series of 40 patients with POAG, PXG and angle recession from the UK underwent the same combined treatment. Patel et $\mathrm{al}^{23}$ reported that after 6 months, IOP decreased by a similar magnitude from 21.1 to $16.7 \mathrm{mmHg}(P<0.01)$, as did the number of drops, from 
2.3 to $0.6(P<0.01)$ as per Table 1 , row 9. Most patients were free of medications in both studies.

A series of 20 patients with cataract and POAG, PXG, or OHT received phacoemulsification with implantation of two iStent inject second-generation devices. ${ }^{24}$ Table 1 , row 11 shows that Arriola-Villalobas et $\mathrm{al}^{24}$ observed an IOP drop of $36 \%(P<0.001)$ from baseline washout and a reduction of one medication $(P<0.001)$, with $75 \%$ of patients discontinuing eye drops at 1 year. Pre- and postoperative endothelial cell count was also measured. In summary, results were positive regardless of the generation of stent, single or double device implantation, and the type of open-angle glaucoma.

Belovay et $\mathrm{al}^{25}$ reported IOP and medication outcomes 1 year after implanting multiple first-generation trabecular stents in combination with phacoemulsification. A total of 53 eyes with mild, moderate, or advanced POAG, PXG, or mixed-mechanism glaucoma were given two or three iStents. In this series, overall IOP decreased from $18.0 \mathrm{mmHg}$ to $14.3 \mathrm{mmHg}(P<0.001)$, without a statistically significant difference $(P=0.76)$ between those who received two versus three stents. Mean medication use dropped by 1.8 and 2.2 agents in the two stent and three stent groups, respectively $(P<0.001)$. Results are summarized in Table 1 , row 5. The authors suggested that the number of stents implanted could be titrated to disease severity and target IOP. ${ }^{25}$

\section{Trabecular stent alone: case series}

One case series from Buchacra et $\mathrm{al}^{26}$ in Spain studied ten phakic and pseudophakic patients with secondary open-angle glaucoma who underwent surgery with a single first-generation iStent without combined phacoemulsification. Secondary causes included steroid-induced glaucoma, traumatic glaucoma, PXG, and PDG. After an average of 12 months, as shown in Table 1, row 10, IOP was lowered by $6.6 \mathrm{mmHg}$ or $27 \%(P<0.05)$ and medications by 1.1 drops $(P<0.05)$. Despite not having cataract surgery, the magnitude of effect from the iStent in this series matched studies with combined procedures. Another case series implanted a single iStent into four eyes, and combined phacoemulsification with an iStent for six eyes, with POAG or PXG. ${ }^{27}$ Highlighted in Table 1, row 8, Vandewalle et $\mathrm{al}^{27}$ observed an overall IOP reduction, with a greater effect in the combined intervention eyes after 12 months.

The effect of the second-generation iStent inject has also been examined in a prospective series without cataract surgery. Voskanyan et al ${ }^{11}$ enrolled 99 phakic and pseudophakic subjects with POAG, PXG, or PDG. At 1 year after implantation of two stents, $66 \%$ achieved IOP $\leq 18$ off medication.
IOP fell $40 \%$, and the majority reduced their medication burden by at least two agents (Table 1, row 12).

\section{Long-term}

The longest reported follow-up period was 5 years in a prospective case series. ${ }^{28}$ A total of 19 subjects with cataract, mild to moderate POAG, PXG, or PDG, and IOP above $18 \mathrm{mmHg}$ underwent cataract surgery with a single iStent. IOP fell $16 \%$ from 19.4 to $16.3 \mathrm{mmHg}(P=0.002)$, which appeared stable after 2 years. Medication use also fell from 1.3 to 0.8 ( $P=0.046)$, with $42 \%$ of subjects off medications entirely (Table 1, row 7). Safety outcomes matched those found in short-term studies, which are discussed in the following section.

\section{Safety}

The first-generation studies reported consistent safety data with few adverse events. Findings were on par with cataract surgery. In the landmark RCT, both groups had equal rates of vitrectomies, postoperative corneal edema, anterior chamber inflammation, IOP elevation, macular edema, and eventual posterior Nd:YAG (neodymium-doped yttrium aluminum garnet) capsulotomies. ${ }^{16,17}$ In both groups, less than $2 \%$ progressed to require a trabeculectomy; over $90 \%$ had corrected distance visual acuity of 20/40 or better and stable visual field parameters at 2 years. ${ }^{16}$ Case series similarly reported good visual outcomes and no episodes of hypotony, choroidal effusions, or flat chambers. ${ }^{21,26,28}$

Most complications related to stent malposition or occlusion early in the postoperative period, affecting $4 \%$ to $18 \%$ of cases. ${ }^{16,19,21,22,25,26}$ These resolved with observation or secondary procedures, including Nd:YAG laser, argon laser gonioplasty, recombinant tissue plasminogen activator, stent repositioning, or stent replacement without negative sequelae. ${ }^{16,17,21,25,26}$ Within the first postoperative week, hyphema also occurred in a couple of case series. ${ }^{23,25,26}$ Intraoperatively, implantation of the stent resulted in iris touch, endothelial touch, or stent malposition less than $10 \%$ of the time. ${ }^{17}$ These events may decrease in frequency with surgeon experience. Steroid response occurred in 2 of 53 eyes 4 and 5 weeks postoperatively in a case series. IOP was controlled after discontinuation of the steroids. ${ }^{25}$ The authors' anecdotal experience suggests that steroid response can be significant with MIGS. We recommend an early taper of steroids in the majority of cases that do not develop significant postoperative inflammation.

Safety outcomes of the iStent inject were reported in two series. Interim analysis of patients who underwent 
phacoemulsification with two implants yielded no loss of vision, hypotony, effusions, or anterior chamber shallowing. ${ }^{24}$ Endothelial cell count loss in these 20 eyes was $13 \%$, although the mean loss of 302.7 cells $/ \mathrm{mm}^{2}$ was accompanied by a high standard deviation of 288.7 cells $/ \mathrm{mm}^{2}{ }^{29}$ In a different study where subjects were implanted with two injects in the absence of cataract surgery, elevated IOP necessitated either medical treatment or surgical intervention in the form of trabeculectomy, goniotrephanation, or deep sclerectomy in less than $5 \%$ within 1 year. ${ }^{11}$ In the same study, the incidence of stent obstruction and malposition was less than 5\%. ${ }^{11}$ Interestingly, one of two of the devices were not visible upon postoperative gonioscopy in 13\%, perhaps because of the small size or use of the original injector, which has since been updated. In a laboratory study of deliberately misplaced first-generation stents, Ichhpujani et $\mathrm{al}^{30}$ demonstrated that ultrasound biomicroscopy could reliably locate the stents, while anterior segment optical coherence tomography and B-scan ultrasonography could not. Future studies are needed to determine whether such imaging modalities can identify second-generation stents not visible under direct gonioscopy.

\section{Other considerations}

\section{Indications}

Trabecular micro-bypass stents are typically indicated for use in conjunction with cataract surgery in adults using ocular hypotensive drugs for mild to moderate open-angle glaucoma. ${ }^{8}$ While the above study populations have included POAG, PXG, PDG, traumatic glaucoma, steroid-induced glaucoma, OHT, and patients without cataract, POAG remains the most common indication. Contraindications include all forms of angle-closure glaucoma and posttrabecular causes of open-angle glaucoma.

No RCTs have examined trabecular micro-bypass stents in advanced open-angle glaucoma. Our opinion is that the use of multiple trabecular bypass stents may work well in more advanced glaucoma disease with reasonable or borderline preoperative IOP control. Furthermore, studies have not assessed the impact of the micro-bypass stent on IOP fluctuation, and so the benefit of the iStent in conditions where IOP fluctuation might play a more important role remains unclear.

\section{Cost}

As with many forms of treatment, cost will continue to play a major role in the uptake of the iStent. To date, one study has examined the cost of trabecular micro-bypass stents compared with other glaucoma treatments. Iordanous et $\mathrm{al}^{31}$ estimated the cumulative cost of two iStents versus medical therapy in Canada. Based on the Ontario Health Insurance Plan, the price of two stents plus disposable intraoperative materials excluding surgeon fees was approximated at Can $\$ 1,044$ per patient. Over 6 years, cost savings of Can\$20.77, Can\$1,272.55, and Can\$2,124.71 per patient were found when comparing two iStents versus monodrug, bidrug, and tridrug therapy, respectively. Similar savings were found with the Trabectome (NeoMedix, Tustin, CA, USA) and endoscopic cyclophotocoagulation compared with medication. The stents plus one medication still showed savings over bidrug or tridrug therapy, but stents with two eye drops were more expensive than tridrug therapy over time. ${ }^{31}$ It appears that the costs of trabecular stents are comparable to and possibly cost saving compared with alternatives in the short term. More data are required to evaluate longterm expenses and cost effectiveness.

\section{Quality of life}

Studies have yet to evaluate the effect of trabecular stents on quality of life. In the case of a successful surgical outcome, we may expect an improvement in quality of life due to reduction or elimination of glaucoma medications. Furthermore, avoiding or delaying the potential complications of more invasive glaucoma surgery may alleviate the negative impact of surgical glaucoma treatment on a patient's quality of life.

\section{Phacoemulsification alone}

The IOP-lowering effect of phacoemulsification alone has been well documented. Long-term retrospective studies have observed reductions of $1-6 \mathrm{mmHg}$ in POAG, normotensive glaucoma, glaucoma suspects, OHT, and normotensive patients without glaucoma. ${ }^{32,33}$ The trabecular micro-bypass RCTs discussed earlier found comparable ranges of IOP reduction in the phacoemulsification control arms. ${ }^{16,17}$ Suggested explanations include improved conventional outflow following a deepened anterior chamber, or decreased aqueous secretion from ciliary body traction via the zonules as the capsule contracts. ${ }^{32}$ What remains unknown is whether the hypotensive effect of phacoemulsification is synergistic with the iStent or is simply additive.

\section{MIGS}

Trabecular micro-bypass stents meet the criteria for MIGS: $\mathrm{ab}$ interno conjunctiva-sparing approach, minimal trauma 
to the target tissue, efficacy, high safety profile, and rapid recovery. ${ }^{6}$ The clear corneal incision spares the conjunctiva for future surgeries if necessary. The ab interno approach allows direct visualization of the angle, optimizing placement of the stents. Schlemm's canal procedures enhance physiologic outflow pathways in the context of normal post-trabecular pathways, thereby reducing IOP and dependency on medication. Furthermore, risks of serious and visually threatening complications associated with other glaucoma surgeries, such as hypotony, choroidal effusions, suprachoroidal hemorrhage, anterior chamber shallowing, and diplopia, are minimized. ${ }^{6}$

Currently, MIGS act in the Schlemm's canal, suprachoroidal space, or subconjunctival space. Devices that improve trabecular flow to Schlemm's canal include the iStent, iStent inject, Trabectome, ${ }^{7,9,10,20,34,35}$ Eyepass (GMP Vision Solutions, Ft Lauderdale, FL, USA; no longer commercially available), ${ }^{36}$ and Excimer laser trabeculotomy (ELT; AIDA, TUI-Laser, Munich, Germany). ${ }^{6,9,35}$ To enhance flow within Schlemm's canal, the Hydrus (Ivantis, Inc., Irvine, CA, USA) ${ }^{20,34,35}$ acts as a canalicular scaffold. To facilitate uveoscleral flow, the CyPass microstent (Transcend Medical, Menlo Park, CA, USA) $)^{20,34}$ or iStent supra (Glaukos Corporation) ${ }^{6}$ are implanted directly into the suprachoroidal space through an ab interno approach. The Aquesys (AqueSys Inc., Aliso Viejo, CA, USA), ${ }^{34}$ which is placed into the subconjunctival space, creates an alternative outflow pathway.

Clinical studies of trabecular devices have yielded successful IOP-lowering outcomes for open-angle glaucoma. The Trabectome, which uses electrocautery to strip the meshwork, has decreased IOP by a range of 4-7 $\mathrm{mmHg}$ and medications by one when combined with phacoemulsification in a number of studies. ${ }^{37-41}$ ELT, which uses photodisruption to create holes in the meshwork, has lowered IOP between 4 and $9 \mathrm{mmHg}$ after 1 year in three studies. ${ }^{42-44}$ While individual treatment effects of some procedures have been compared with phacoemulsification, no studies have directly compared MIGS devices.

\section{Conclusion}

MIGS with the trabecular micro-bypass stent has been shown in numerous publications and large-scale studies to be a safe and effective procedure for mild to moderate open-angle glaucoma. The supportive evidence favoring the risk-benefit ratio of iStent implantation is most clear for concurrent phacoemulsification and iStent implantation in patients with mild to moderate open-angle glaucoma. Indications may expand to include patients with OHT, advanced glaucoma, and secondary open-angle glaucomas as more evidence becomes available for these conditions.

\section{Disclosure}

Dr Hady Saheb has received travel funding from Glaukos. The authors report no other conflicts of interest in this work.

\section{References}

1. Quigley HA, Broman AT. The number of people with glaucoma worldwide in 2010 and 2020. Br J Ophthalmol. 2006;90(3):262-267.

2. Leung EW, Medeiros FA, Weinreb RN. Prevalence of ocular surface disease in glaucoma patients. J Glaucoma. 2008;17(5):350-355.

3. Gupta R, Patil B, Shah BM, Bali SJ, Mishra SK, Dada T. Evaluating eye drop instillation technique in glaucoma patients. J Glaucoma. 2012;21(3):189-192.

4. Adio AO, Onua AA. Economic burden of glaucoma in Rivers State, Nigeria. Clin Ophthalmol. 2012;6:2023-2031.

5. Gedde SJ, Herndon LW, Brandt JD, et al; Tube Versus Trabeculectomy Study Group. Postoperative complications in the Tube Versus Trabeculectomy (TVT) study during five years of follow-up. Am J Ophthalmol. 2012;153(5):804-814.e1.

6. Saheb H, Ahmed II. Micro-invasive glaucoma surgery: current perspectives and future directions. Curr Opin Ophthalmol. 2012; 23(2):96-104.

7. Razeghinejad MR, Spaeth GL. A history of the surgical management of glaucoma. Optom Vis Sci. 2011;88(1):E39-E47.

8. Francis BA, Winarko J. Ab interno Schlemm's canal surgery: trabectome and iStent. Dev Ophthalmol. 2012;50:125-136.

9. Francis BA, Singh K, Lin SC, et al. Novel glaucoma procedures: a report by the American Academy of Ophthalmology. Ophthalmology. 2011;118(7):1466-1480.

10. Minckler DS, Hill RA. Use of novel devices for control of intraocular pressure. Exp Eye Res. 2009;88(4):792-798.

11. Voskanyan L, García-Feijoó J, Belda JI, et al; Synergy Study Group. Prospective, unmasked evaluation of the iStent ${ }^{\mathbb{}}$ inject system for open-angle glaucoma: synergy trial. Adv Ther. 2014;31(2): 189-201.

12. Bahler CK, Hann CR, Fjield T, Haffner D, Heitzmann H, Fautsch MP. Second-generation trabecular meshwork bypass stent (iStent inject) increases outflow facility in cultured human anterior segments. Am J Ophthalmol. 2012;153(6):1206-1213.

13. Goel M, Picciani RG, Lee RK, Bhattacharya SK. Aqueous humor dynamics: a review. Open Ophthalmol J. 2010;4:52-59.

14. Hunter KS, Fjield T, Heitzmann H, Shandas R, Kahook MY. Characterization of micro-invasive trabecular bypass stents by ex vivo perfusion and computational flow modeling. Clin Ophthalmol. 2014;8:499-506.

15. Bahler CK, Smedley GT, Zhou J, Johnson DH. Trabecular bypass stents decrease intraocular pressure in cultured human anterior segments. Am J Ophthalmol. 2004;138(6):988-994.

16. Craven ER, Katz LJ, Wells JM, Giamporcaro JE; iStent Study Group. Cataract surgery with trabecular micro-bypass stent implantation in patients with mild-to-moderate open-angle glaucoma and cataract: two-year follow-up. J Cataract Refract Surg. 2012;38(8): $1339-1345$.

17. Samuelson TW, Katz LJ, Wells JM, Duh YJ, Giamporcaro JE; US iStent Study Group. Randomized evaluation of the trabecular micro-bypass stent with phacoemulsification in patients with glaucoma and cataract. Ophthalmology. 2011;118(3):459-467.

18. Fea AM. Phacoemulsification versus phacoemulsification with micro-bypass stent implantation in primary open-angle glaucoma: randomized double-masked clinical trial. J Cataract Refract Surg. 2010;36(3):407-412. 
19. Fernández-Barrientos Y, García-Feijoó J, Martínez-de-la-Casa JM, Pablo LE, Fernández-Pérez C, García Sánchez J. Fluorophotometric study of the effect of the glaukos trabecular microbypass stent on aqueous humor dynamics. Invest Ophthalmol Vis Sci. 2010;51(7):3327-3332.

20. Augustinus CJ, Zeyen T. The effect of phacoemulsification and combined phaco/glaucoma procedures on the intraocular pressure in openangle glaucoma. A review of the literature. Bull Soc Belge Ophtalmol. 2012;(320):51-66.

21. Spiegel D, García-Feijoó J, García-Sánchez J, Lamielle H. Coexistent primary open-angle glaucoma and cataract: preliminary analysis of treatment by cataract surgery and the iStent trabecular micro-bypass stent. Adv Ther. 2008;25(5):453-464.

22. Spiegel D, Wetzel W, Neuhann T, et al. Coexistent primary open-angle glaucoma and cataract: interim analysis of a trabecular microbypass stent and concurrent cataract surgery. Eur J Ophthalmol. 2009;19(3):393-399.

23. Patel I, de Klerk TA, Au L. Manchester iStent study: early results from a prospective UK case series. Clin Experiment Ophthalmol. 2013;41(7):648-652.

24. Arriola-Villalobos P, Martínez-de-la-Casa JM, Díaz-Valle D, et al. Midterm evaluation of the new Glaukos iStent with phacoemulsification in coexistent open-angle glaucoma or ocular hypertension and cataract. Br J Ophthalmol. 2013;97(10):1250-1255.

25. Belovay GW, Nagi A, Chan BJ, Rateb M, Ahmed II. Using multiple trabecular micro-bypass stents in cataract patients to treat open-angle glaucoma. J Cataract Refract Surg. 2012;38(11):1911-1917.

26. Buchacra O, Duch S, Milla E, Stirbu O. One-year analysis of the iStent trabecular microbypass in secondary glaucoma. Clin Ophthalmol. 2011;5:321-326.

27. Vandewalle E, Zeyen T, Stalmans I. The iStent trabecular micro-bypass stent: a case series. Bull Soc Belge Ophtalmol. 2009;(311):23-29.

28. Arriola-Villalobos P, Martínez-de-la-Casa JM, Díaz-Valle D, Fernández-Pérez C, García-Sánchez J, García-Feijoó J. Combined iStent trabecular micro-bypass stent implantation and phacoemulsification for coexistent open-angle glaucoma and cataract: a long-term study. $\mathrm{Br} \mathrm{J}$ Ophthalmol. 2012;96(5):645-649.

29. Park J, Yum HR, Kim MS, Harrison AR, Kim EC. Comparison of phaco-chop, divide-and-conquer, and stop-and-chop phaco techniques in microincision coaxial cataract surgery. J Cataract Refract Surg. 2013;39(10):1463-1469.

30. Ichhpujani P, Katz LJ, Gille R, Affel E. Imaging modalities for localization of an iStent ${ }^{\circledR}$. Ophthalmic Surg Lasers Imaging. 2010;41(6):660-663.

31. Iordanous Y, Kent JS, Hutnik CM, Malvankar-Mehta MS. Projected cost comparison of Trabectome, iStent, and endoscopic cyclophotocoagulation versus glaucoma medication in the Ontario Health Insurance Plan. J Glaucoma. 2014;23(2):e112-e118.
32. Shingleton BJ, Pasternack JJ, Hung JW, O’Donoghue MW. Three and five year changes in intraocular pressures after clear corneal phacoemulsification in open angle glaucoma patients, glaucoma suspects, and normal patients. J Glaucoma. 2006;15(6):494-498.

33. Poley BJ, Lindstrom RL, Samuelson TW. Long-term effects of phacoemulsification with intraocular lens implantation in normotensive and ocular hypertensive eyes. J Cataract Refract Surg. 2008;34(5):735-742.

34. Brandão LM, Grieshaber MC. Update on Minimally Invasive Glaucoma Surgery (MIGS) and new implants. J Ophthalmol. 2013;2013:705915.

35. Kaplowitz K, Schuman JS, Loewen NA. Techniques and outcomes of minimally invasive trabecular ablation and bypass surgery. $\mathrm{Br} J$ Ophthalmol. 2014;98(5):579-585.

36. Schmidt W, Kastner C, Sternberg K, et al. New concepts for glaucoma implants: controlled aqueous humor drainage, encapsulation prevention and local drug delivery. Curr Pharm Biotechnol. 2013;14(1):98-111.

37. Ting JL, Damji KF, Stiles MC; Trabectome Study Group. Ab interno trabeculectomy: outcomes in exfoliation versus primary open-angle glaucoma. J Cataract Refract Surg. 2012;38(2):315-323.

38. Francis BA, Minckler D, Dustin L, et al; Trabectome Study Group. Combined cataract extraction and trabeculotomy by the internal approach for coexisting cataract and open-angle glaucoma: initial results. J Cataract Refract Surg. 2008;34(7):1096-1103.

39. Minckler D, Mosaed S, Dustin L, Ms BF; Trabectome Study Group. Trabectome (trabeculectomy-internal approach): additional experience and extended follow-up. Trans Am Ophthalmol Soc. 2008;106:149-159; discussion 159-160.

40. Jordan JF, Wecker T, van Oterendorp C, et al. Trabectome surgery for primary and secondary open angle glaucomas. Graefes Arch Clin Exp Ophthalmol. 2013;251(12):2753-2760.

41. Ahuja Y, Ma Khin Pyi S, Malihi, Hodge DO, Sit AJ. Clinical results of $\mathrm{ab}$ interno trabeculotomy using the trabectome for open-angle glaucoma: the Mayo Clinic series in Rochester, Minnesota. Am J Ophthalmol. 2013;156(5):927-935.e2.

42. Töteberg-Harms M, Hanson JV, Funk J. Cataract surgery combined with excimer laser trabeculotomy to lower intraocular pressure: effectiveness dependent on preoperative IOP. BMC Ophthalmol. 2013;13:24.

43. Töteberg-Harms M, Ciechanowski PP, Hirn C, Funk J. [One-year results after combined cataract surgery and excimer laser trabeculotomy for elevated intraocular pressure]. Ophthalmologe. 2011;108(8):733-738. German [with English abstract].

44. Wilmsmeyer S, Philippin H, Funk J. Excimer laser trabeculotomy: a new, minimally invasive procedure for patients with glaucoma. Graefes Arch Clin Exp Ophthalmol. 2006;244(6):670-676.
Clinical Ophthalmology

\section{Publish your work in this journal}

Clinical Ophthalmology is an international, peer-reviewed journal covering all subspecialties within ophthalmology. Key topics include: Optometry; Visual science; Pharmacology and drug therapy in eye diseases; Basic Sciences; Primary and Secondary eye care; Patient Safety and Quality of Care Improvements. This journal is indexed on Submit your manuscript here: http://www.dovepress.com/clinical-ophthalmology-journal

\section{Dovepress}

PubMed Central and CAS, and is the official journal of The Society of Clinical Ophthalmology (SCO). The manuscript management system is completely online and includes a very quick and fair peer-review system, which is all easy to use. Visit http://www.dovepress.com/ testimonials.php to read real quotes from published authors. 